\title{
Can programmatic inputs improve adolescent mothers' access to care in rural Bangladesh? Nine- years of evidence from a cohort study
}

\section{CURRENT STATUS: UNDER REVIEW}

Journal of Health, Population and Nutrition $\triangle B M C$

Aminur Rahman

International Centre for Diarrhoeal Disease Research

$\checkmark$ draminur@icddrb.orgCorresponding Author

ORCiD: https://orcid.org/0000-0003-1434-3883

Tahmina Begum

International Centre for Diarrhoeal Disease Research Bangladesh

Anne Austin

JSI Research and Training Institute Inc

Md. Hasan

Bangabandhu Sheikh Mujib Medical University

Nurul Alam

International Centre for Diffraction Data

Iqbal Anwar

International Centre for Diarrhoeal Disease Research Bangladesh

Surasak Taneepanichskul

Chulalongkorn University College of Public Health Sciences

DOI:

$10.21203 / \mathrm{rs} .2 .21953 / \mathrm{v} 1$

SUBJECT AREAS

Nutrition \& Dietetics

KEYWORDS

Adolescent pregnancy, Antenatal Care, Facility Delivery, Maternal Health, Bangladesh 
Abstract

Background Adolescent mothers (Girls aged 15-19) constitute $8 \%$ of annual global births, but account for $10 \%$ of annual maternal deaths. WHO recommended 4-8 Antenatal Care (ANC) visits, in addition to quality care and facility-based deliveries, are well-documented interventions to reduce maternal and child morbidity and mortality. Determinants of maternal and child health care in Bangladesh have received considerable attention, but less attention has been focused on adolescent mothers. This study explores the factors associated with 4 or more (4+) ANC visits and facility-based delivery among adolescent mothers in one rural area of Bangladesh,

Methods This study uses Health and Demographic Surveillance System (HDSS) data. We conducted a comparative study on trends in 4+ ANC visits and facility-based deliveries among adolescent mothers (10-19 years) residing in an intervention area (icddr, b service area, ISA) against a comparison area (government service areas, GSA) of HDSS between 2007 and 2015. 4,996 adolescent mothers were included in the final analysis. Binary logistic regression was used to document the statistical difference on outcome indicators in the two study areas.

Results Trends in 4+ANC visits and facility-based deliveries were higher in the ISA relative to the GSA. The adjusted odds of an adolescent mother accessing 4+ ANC visits in the GSA, relative to ISA was 0.57 (95\% Cl: $0.49-0.66, \mathrm{p}$-value <0.05); the adjusted odds of an adolescent mother accessing facility-based delivery in the ISA, relative to GSA was 6.63 (95\% Cl: $5.85-7.52$, p-value <0.05). Increasing numbers of ANC visits were associated with increases in facility-based births in both the ISA and GSA.

Conclusion This study documented that both 4+ ANC visits and facility delivery rates among adolescent mothers are much higher in the ISA than GSA. Increasing 4+ ANC visits and facility deliveries over the years, particularly in the ISA, coincide with programmatic efforts to improve the quality and availability of maternal and newborn health services. Learning from existing interventions in ISA and applying them to other areas will strengthen Bangladesh's efforts to improve maternal and newborn health outcomes and achieve the sustainable development goal 3 (SDG 3). Full-text 
Due to technical limitations, full-text HTML conversion of this manuscript could not be completed. However, the manuscript can be downloaded and accessed as a PDF.

Figures

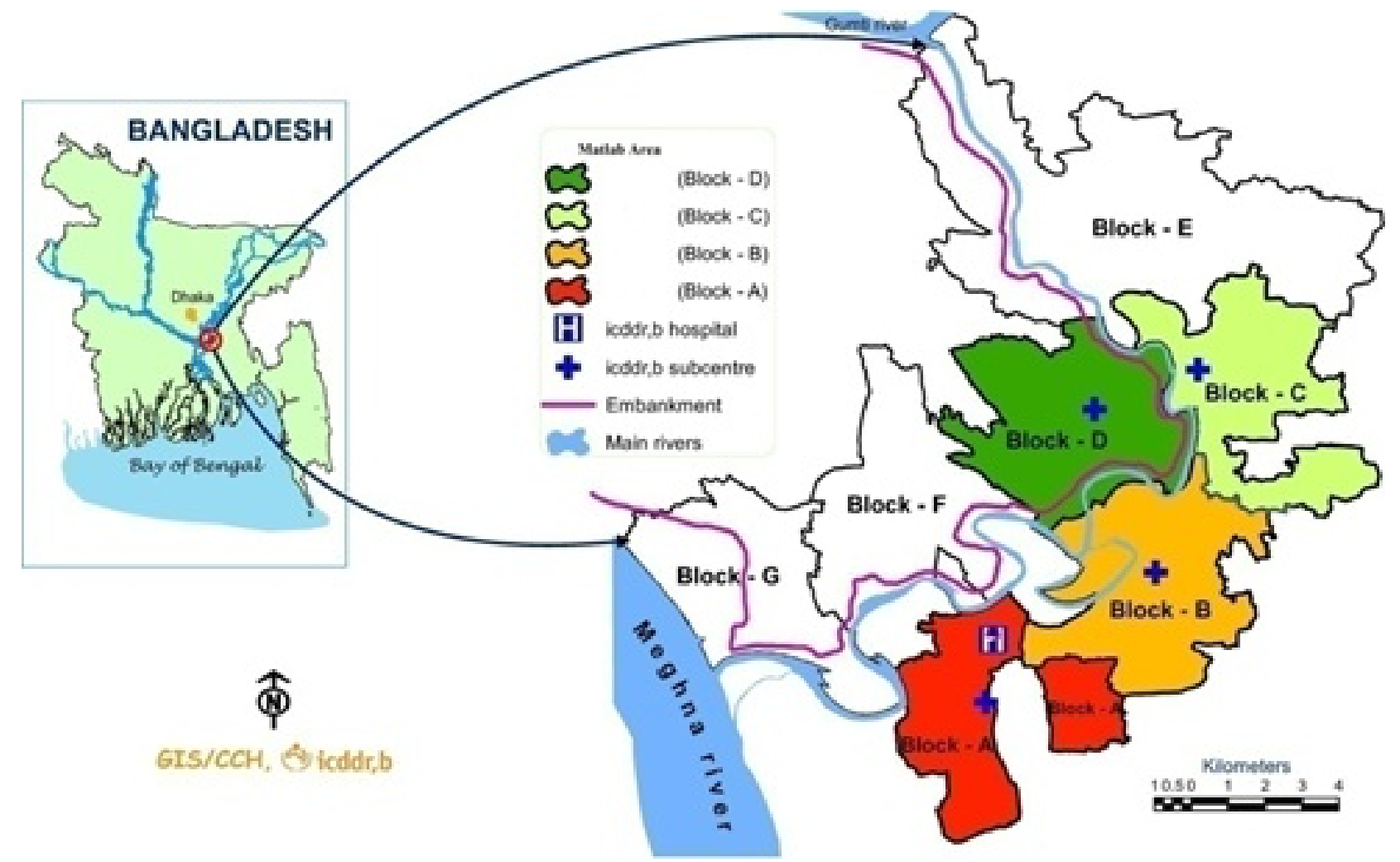

Figure 1

Matlab Study setting 


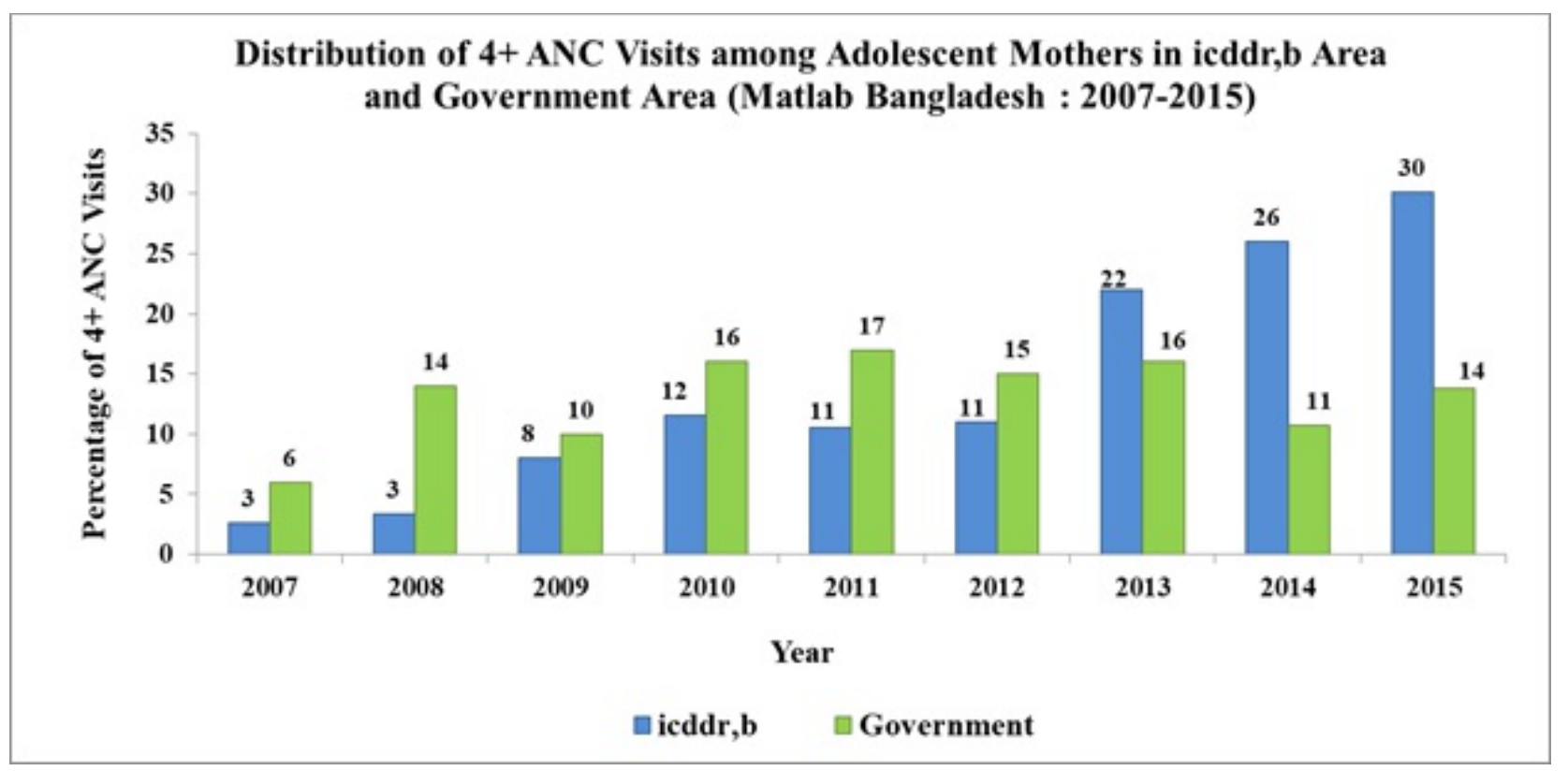

Figure 2

Distribution of 4+ ANC visits in both icddr,b service area and Government service area among adolescent mothers (Matlab Bangladesh: 2007-2015).

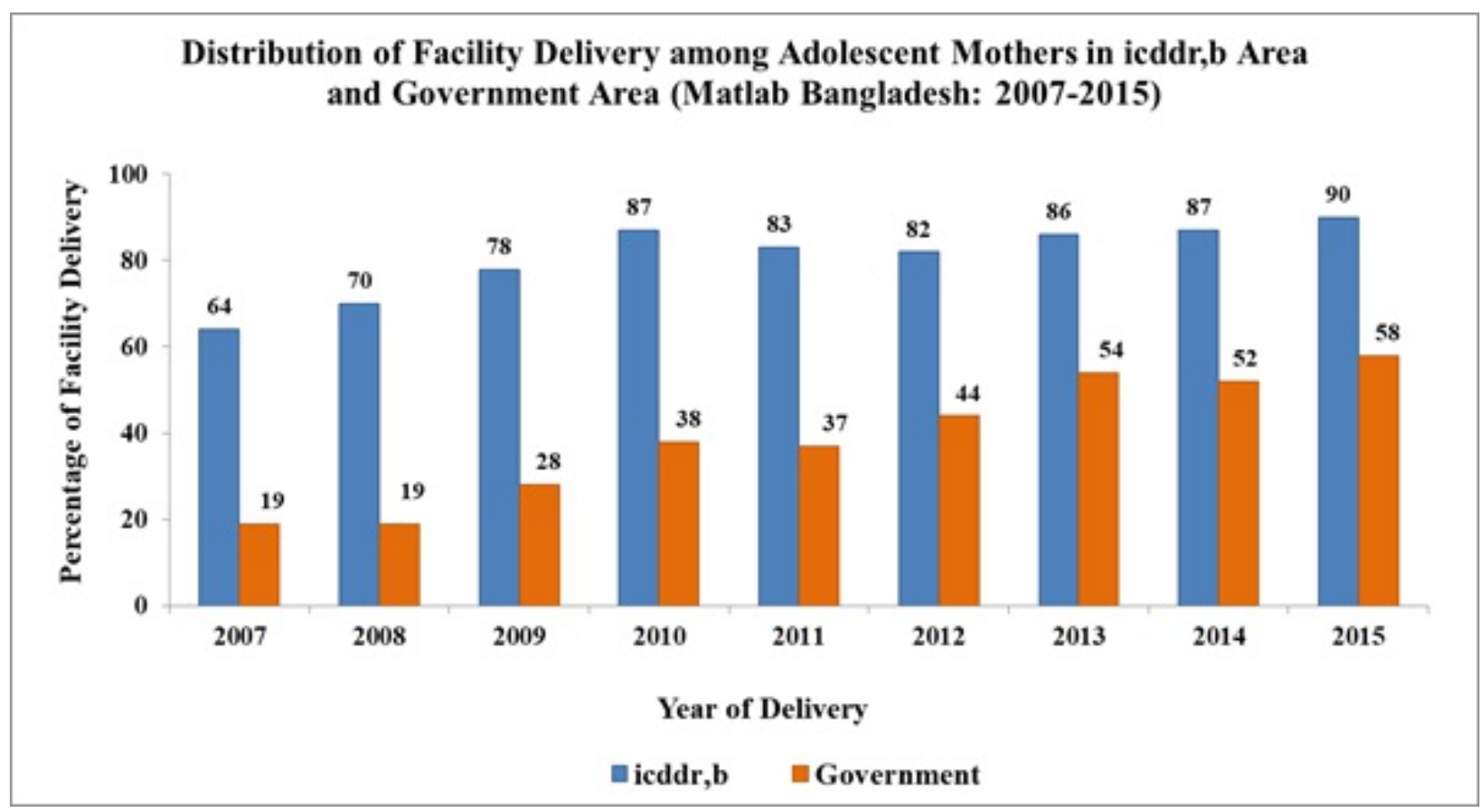

Figure 3

Distribution of Facility Delivery among adolescent mothers in icddr, b service area and Government service area (Matlab Bangladesh: 2007-2015) 